\title{
Tumor vasoproliferativo associado à tuberculose ocular presumida: relato de caso
}

\author{
Vasoproliferative tumor associated with presumed ocular tuberculosis:case report
}

\author{
Luciana Duarte Rodrigues ${ }^{1}$ \\ Luciana Lucci Serracarbassa ${ }^{2}$ \\ Haley Rosa ${ }^{3}$ \\ Yoshitaka Nakashima ${ }^{4}$ \\ Pedro Durães Serracarbassa ${ }^{5}$
}

\begin{tabular}{|c|}
\hline RESUMO \\
\hline 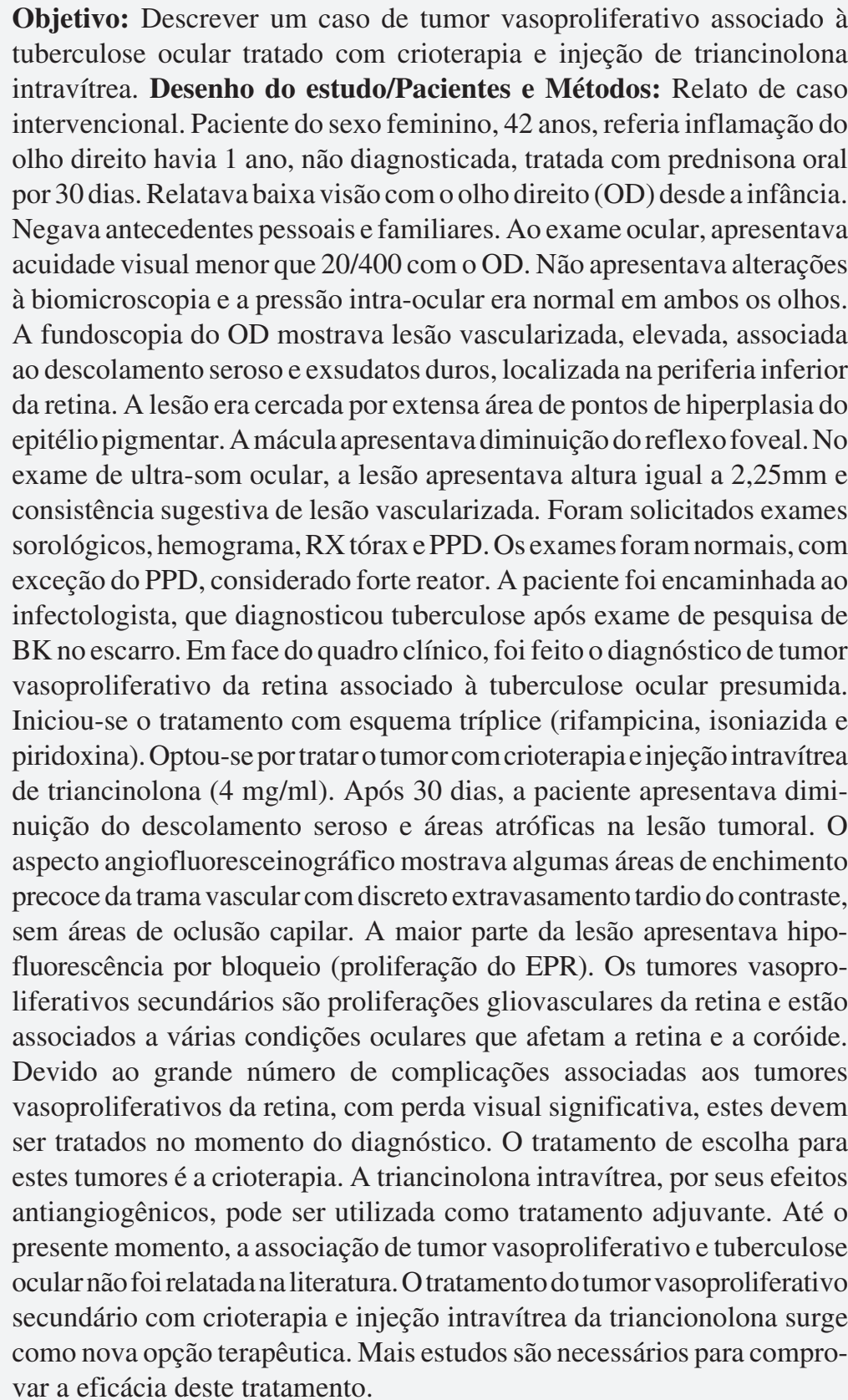 \\
\hline
\end{tabular}

Descritores: Neoplasias da retina/diagnóstico; Neovascularização retiniana; Tuberculose ocular; Crioterapia; Triancinolona/administração \& dosagem; Relato de casos [Tipo de publicação]
Trabalho realizado na Disciplina de Oftalmologia do Hospital das Clínicas da Universidade de São Paulo - USP São Paulo (SP) - Brasil.

Pós-graduanda em Medicina pela Faculdade de Medicina da Universidade de São Paulo - USP - São Paulo (SP) - Brasil.

Colaboradora do Departamento de Oftalmologia da Santa Casa de Misericórdia de São Paulo - São Paulo (SP) Brasil.

Estagiário do Setor de Uveíte do Hospital das Clínicas da Faculdade de Medicina da USP - São Paulo (SP) - Brasil. ${ }^{4}$ Doutor em Medicina; Médico Assistente do Setor de Retina do Hospital das Clínicas da Faculdade de Medicina da USP - São Paulo (SP) - Brasil.

5 Doutor em Medicina; Médico Colaborador do Setor de Retina do Hospital das Clínicas da Faculdade de Medicina da USP - São Paulo (SP) - Brasil.

Endereço para correspondência: Pedro D. Serracarbassa. Av. Brigadeiro Faria Lima, 1903 - Conj. 43 - São Paulo (SP) CEP 01472-001

E-mail: serracar@brfree.com.br

Recebido para publicação em 02.03.2006

Última versão recebida em 09.10.2006

Aprovação em 25.10.2006 


\section{INTRODUÇÃO}

Os tumores vasoproliferativos da retina são lesões benignas e relativamente raras, inicialmente descritas por Shields ${ }^{(1)}$ em 1983 e posteriormente classificadas como tumores vasoproliferativos primários e secundários, de acordo com sua etiologia ${ }^{(2)}$. Os tumores primários apresentam-se como massas vasculares róseo-alaranjadas que acometem a retina sensorial, caracteristicamente unilaterais, solitárias e geralmente localizadas no quadrante temporal inferior. Observam-se também vasos nutridores e de drenagem, não dilatados, direcionados para o tumor, além de exsudação ao redor da lesão, hemorragias retinianas, descolamento exsudativo da retina e hemorragia vítrea. O aspecto angiofluoresceinográfico mostra enchimento precoce da trama vascular com áreas de extravasamento tardio do contraste, sem áreas de oclusão capilar. Não há história familiar ou doenças associadas. Os tumores secundários estão associados a várias condições oculares que afetam a retina e a coróide, como uveítes, retinocoroidites, doença falciforme, retinose pigmentar, retinopatia da prematuridade e descolamentos antigos da retina. As lesões são menores e associadas a áreas de proliferação do EPR. No presente estudo, relata-se um caso de tumor vasoproliferativo da retina associado à tuberculose ocular presumida e discutem-se aspectos de seu tratamento. Até o presente momento, a associação de tumor vasoproliferativo e tuberculose ocular não foi relatada na literatura.

\section{RELATO DO CASO}

Paciente MS, 42 anos, sexo feminino, natural de Santos. Referia inflamação do olho direito há 1 ano atrás, não diagnosticada, tratada com prednisona oral por 30 dias. Relatava ainda baixa visão no olho direito desde a infância. Negava antecedentes pessoais e familiares. Ao exame ocular, apresentava acuidade visual menor que 20/400 no OD e 20/20 no OE. Não apresentava alterações à biomicroscopia e a pressão ocular era normal em ambos os olhos. A fundoscopia do OD mostrava lesão vascularizada, elevada, associada ao descolamento seroso e exsudatos duros, localizada na periferia inferior da retina. A lesão ocupava aproximadamente 2 horas e era cercada por extensa área de pontos de hiperplasia do epitélio pigmentar. A mácula apresentava diminuição do brilho foveal. No exame de ultra-som ocular, a lesão apresentava altura igual a $2,25 \mathrm{~mm}$ e consistência sugestiva de lesão vascularizada. Foram solicitados os seguintes exames subsidiários: hemograma, sorologia para toxoplasmose e lues, RX tórax e PPD. Os exames foram normais, com exceção do PPD, considerado forte reator. A paciente foi então encaminhada ao infectologista, que diagnosticou tuberculose após exame de pesquisa de BK no escarro. Frente ao quadro clínico, foi feito o diagnóstico de tumor vasoproliferativo da retina associado à tuberculose ocular presumida. Iniciou-se então o tratamento com esquema tríplice (rifampicina, isoniazida e piridoxina). Optou-se por tratar o tumor através da crioterapia e injeção intravítrea de triancinolona. Após 30 dias, a paciente apresentava diminuição do descolamento seroso e áreas atróficas na lesão tumoral. $\mathrm{O}$ aspecto angiofluoresceinográfico mostrava algumas áreas de enchimento precoce da trama vascular com discreto extravasamento tardio do contraste, sem áreas de oclusão capilar. A maior parte da lesão apresentava hipofluorescência por bloqueio (proliferação do EPR).

\section{DISCUSSÃO}

Apenas recentemente os tumores vasoproliferativos foram definidos como uma entidade distinta entre os tumores intraoculares $^{(1,3-5)}$. A patogênese destes tumores ainda não foi esclarecida. A histologia revelou tratar-se de lesões benignas compostas por proliferação gliovascular reativa ${ }^{(3)}$. A extensão variável de proliferação vascular e gliose ocasionou uma variedade de termos similares na literatura, como lesão hemangioma-like ${ }^{(6)}$, lesão angioma-like ${ }^{(7)}$, massa angiomatosa ${ }^{(8)}$, telangiectasias retinianas periféricas ${ }^{(9)}$ e hemangiomas retinianos adquiridos ${ }^{(1)}$.

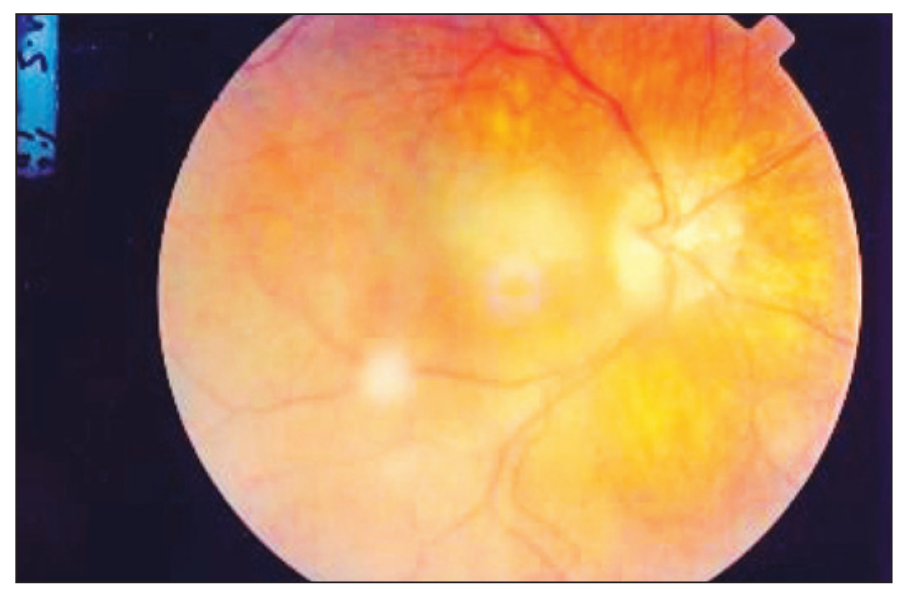

Figura 1 - Aspecto do pólo posterior, 1 ano após a inflamação ocular referida

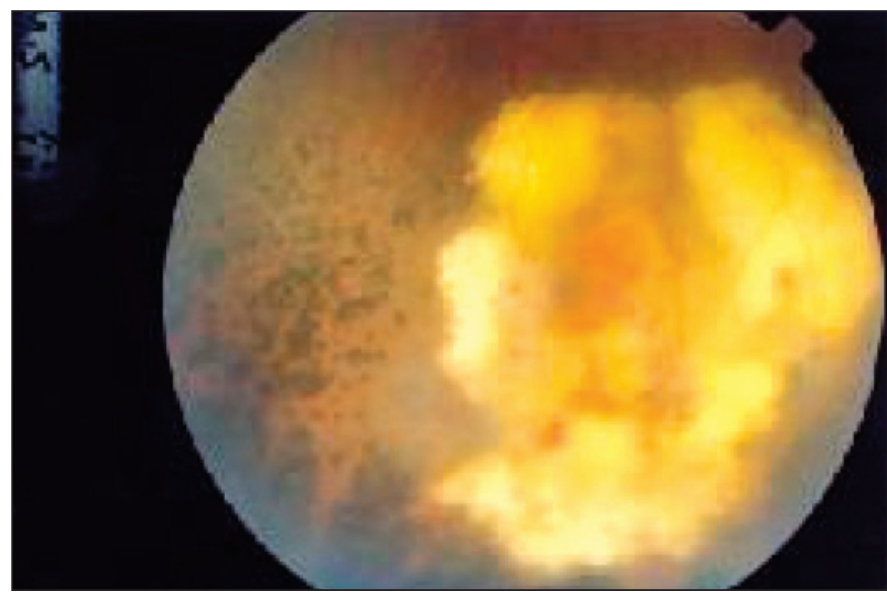

Figura 2 - Tumor vasoproliferativo associado ao descolamento seroso, exsudação lipídica e proliferação do EPR 
Acredita-se que estas lesões sejam causadas por uma variedade de processos que promovem proliferação vascular e do epitélio pigmentar. Os fatores liberados localmente durante a quebra da barreira hemato-retiniana que ocorre, por exemplo, nas uveítes, seriam responsáveis pela proliferação de células gliais, com vasoproliferação secundária ${ }^{(10-11)}$.

Os tumores vasoproliferativos são classificados como primários ou secundários de acordo com a sua etiologia ${ }^{(2)}$. Os tumores primários tendem a ser solitários, pequenos e localizados na ora serrata, geralmente no quadrante temporal inferior. Não há história familiar ou doenças associadas ${ }^{(2)}$. Os tumores secundários estão associados a várias condições oculares que afetam a retina e a coróide, como uveítes, retinocoroidites, doença falciforme, retinose pigmentar, retinopatia da prematuridade e descolamentos antigos da retina ${ }^{(2)}$. As lesões são menores e associadas a áreas de proliferação do $\mathrm{EPR}^{(2)}$. No presente estudo, a paciente apresentou um tumor vasoproliferativo da retina associado à tuberculose ocular presumida. A

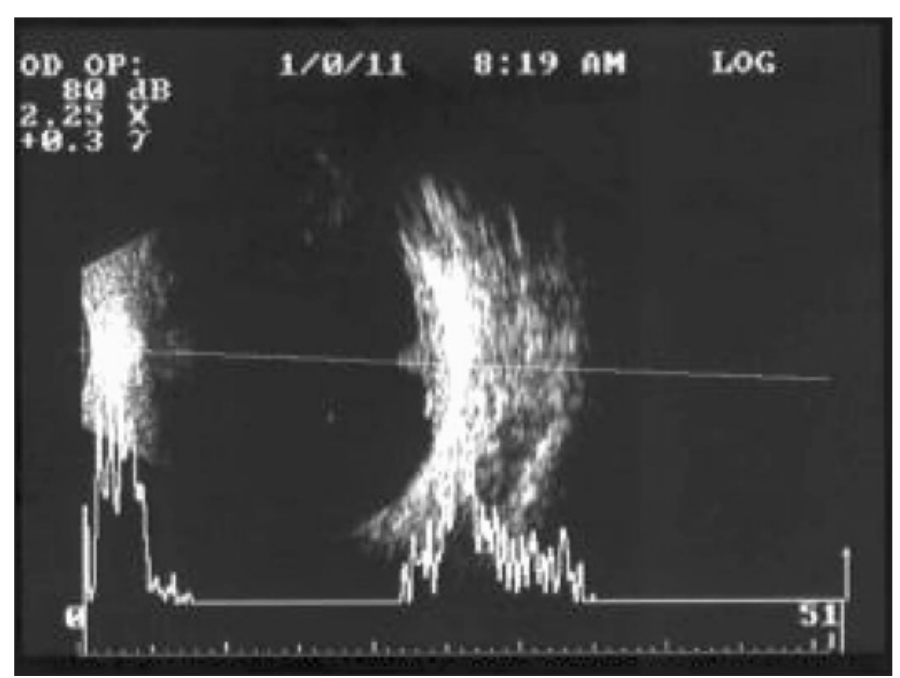

Figura 3 - Ultra-som ocular: lesão vascularizada, 2,25 mm

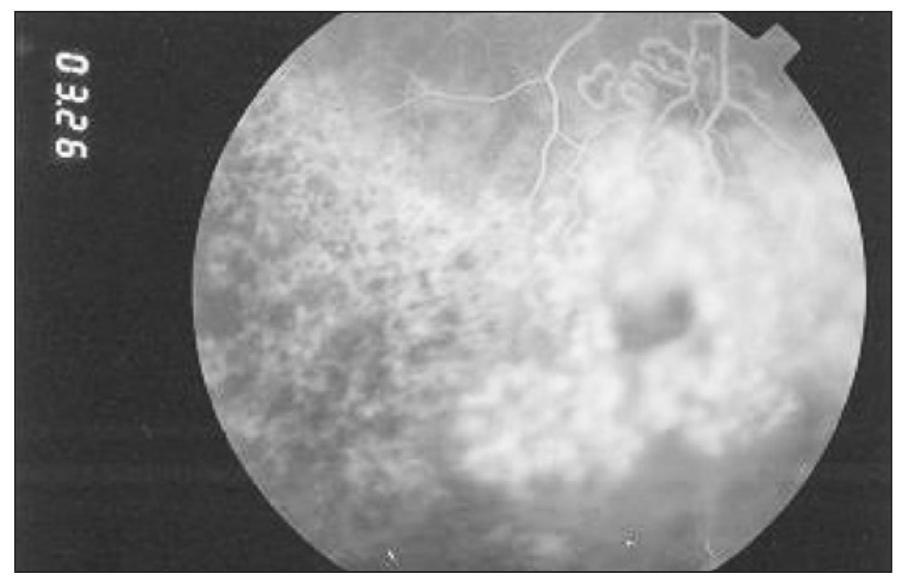

Figura 4 - Angiofluoresceinografia: enchimento vascular precoce, extravasamento discreto e hipofluorescência por bloqueio (EPR) paciente referia inflamação do olho direito há 1 ano atrás, não diagnosticada, tratada com prednisona oral por 30 dias. O tumor vasoproliferativo foi um achado de exame, pois a paciente não apresentava queixas e o tumor não apresentava exsudação ou descolamento seroso importante nem associação com hemorragias. Após exame de pesquisa de BK no escarro, foi feito o diagnóstico de tuberculose.

Os diagnósticos diferenciais incluem as lesões tumorais e vasculares, entre elas o hemangioma capilar da síndrome de von Hippel-Lindau, a doença de Coats e coriorretinopatia hemorrágica exsudativa periférica. Além dessas, devem ser considerados os tumores malignos que podem afetar a retina, como as metástases na coróide e retina e os melanomas da coróide.

Os hemangiomas capilares retinianos na síndrome de von Hippel-Lindau ocorrem em pacientes mais jovens, são múltiplos, bilaterais, também possuem vasos nutridores e de drenagem, localizados em quaisquer quadrantes da retina ${ }^{(12)}$. Duas características que os diferenciam dos tumores vasoproliferativos são a importante dilatação dos vasos nutridores e a exsudação presente na mácula, que confere o seu aspecto de "mácula em estrela"(11). Os pacientes apresentam características clínicas da síndrome e história familiar positiva. No caso relatado, a paciente apresentava tortuosidade sem dilatação dos vasos nutridores e sem exsudação macular. A lesão era unilateral. Não apresentava nenhuma característica da síndrome ou história familiar.

A doença de Coats acomete crianças e jovens do sexo masculino; as lesões são menores e presentes desde o nascimento. Pode haver dilatação telangiectásica dos vasos da retina, exsudação e descolamentos da retina, como ocorre nos tumores vasoproliferativos. Entretanto, as lesões apresentam acúmulo de exsudato lipídico na doença de Coats e os descolamentos da retina são extensos ${ }^{(13)}$. No presente estudo, a paciente pertencia a uma faixa etária maior, era mulher e a lesão não apresentava exsudatos lipídicos ou descolamento extenso.

Os melanomas amelanocíticos e as metástases sistêmicas acometem a coróide, mas pode haver metástase na própria retina ou infiltração da coróide ${ }^{(14)}$. Nos casos em que o diagnóstico diferencial não pode ser feito pelo exame clínico ou por angiofluoresceinografia e ultra-som, há indicação de biópsia da lesão por vitrectomia via pars plana ${ }^{(11)}$. No caso relatado, o exame de ultra-som ocular era sugestivo de lesão vascularizada, mas sem sinais típicos do melanoma. $\mathrm{O}$ aspecto angiofluoresceinográfico mostrava algumas áreas de enchimento precoce da trama vascular com discreto extravasamento tardio do contraste, sem áreas de oclusão capilar. A maior parte da lesão apresentava hipofluorescência por bloqueio (proliferação do EPR).

Outro diferencial é a coriorretinopatia hemorrágica exsudativa periférica, que cursa com hemorragias periféricas retinianas, sub-retinianas e hemorragias vítreas, além de lesões tumorais, exsudatos e descolamentos exsudativos. $\mathrm{O}$ acometimento é bilateral, em pacientes acima de 60 anos e há associação com degeneração macular. Os tumores são localizados no quadrante temporal inferior, são geralmente sub-retinianos e 
apresentam alterações pigmentares secundárias a hemorragia ${ }^{(15)}$. Não há conexão entre os vasos do tumor e a retina, ao contrário do que ocorre nos tumores vasoproliferativos ${ }^{(15)}$. No caso da paciente em questão, a lesão apresentava vasos nutridores e de drenagem, não dilatados, direcionados para o tumor.

A história natural dos tumores vasoproliferativos é bastante variável. Pode haver involução ou progressão lenta ${ }^{(5,9)}$. Por outro lado, como a maioria dos pacientes apresenta lesões sintomáticas e os sintomas estão geralmente associados ao aumento da exsudação, é provável que ocorra progressão da doença e acometimento visual secundário na maioria dos $\operatorname{casos}^{(11)}$.

Além disso, apesar da localização periférica, estes tumores podem comprometer a acuidade visual por causa das alterações vítreo-retinianas associadas, como exsudação sub ou intra-retiniana, líquido sub-retiniano, hemorragia vítrea, fibrose macular pré-retiniana e edema macular ${ }^{(1,4,16)}$.

As hemorragias sub, intra e pré-retinianas são causadas por exsudação ou mesmo rotura dos vasos do tumor ou vasos adjacentes e podem variar em severidade, causando menor ou maior baixa visual ${ }^{(1,4,6)}$. No caso relatado, a paciente apresentava baixa visão no olho afetado desde a infância e a lesão não apresentava exsudação importante ou sinais de hemorragia vítrea. Entretanto, devido ao grande número de complicações associadas aos tumores vasoproliferativos da retina, com perda visual significativa, recomenda-se que estes sejam tratados no momento do diagnóstico ${ }^{(9)}$.

O tratamento de escolha para estes tumores é a crioterapia, com resultados satisfatórios, sendo a fotocoagulação e a vitrectomia reservadas para situações específicas ${ }^{(1,6,16-17)}$.

A crioterapia é indicada para tumores pequenos, com menos de $2 \mathrm{~mm}$. Nos tumores maiores, além da dificuldade de tratar toda a espessura da lesão, existe ainda a possibilidade de aumentar a exsudação e o descolamento associados ${ }^{(1,6)}$. Mesmo nos tumores menores, a crioterapia isolada pode não ser suficiente ${ }^{(4)}$.

A radioterapia pode ser usada em lesões com $2 \mathrm{~mm}$ ou mais, com bons resultados, usando braquiterapia com placas de rutênio $106^{(18-19)}$. A vantagem da técnica é a possibilidade de tratar toda a lesão, até o seu ápice, com relativa segurança devido à sua localização periférica. Hemorragia e exsudação secundárias ao dano direto à lesão que ocorre na crioterapia e na fotocoagulação são menos freqüentes ${ }^{(18-19)}$. Porém, a dose ideal para o tratamento dos tumores vasoproliferativos ainda não foi estabelecida. Outra desvantagem são as complicações como catarata e retinopatia por irradiação ${ }^{(20)}$.

A terapia fotodinâmica com verteporfina também foi utilizada no tratamento do tumor vasoproliferativo, permitindo o tratamento seletivo do tumor neovascular ${ }^{(21-22)}$. A dose ideal também não foi estabelecida, observando-se recidivas com doses iguais a $50 \mathrm{~J} / \mathrm{cm}^{2(21)} \mathrm{e}$ aumento da exsudação com doses iguais a $100 \mathrm{~J} / \mathrm{cm}^{2(22)}$.

Os corticóides apresentam atividade antiangiogênica e antiinflamatória, além de efeitos sobre a permeabilidade vascular $^{(23-26)}$, porém o seu modo de ação sobre as células endoteliais da coróide ainda não está definido. Acredita-se que a triancinolona seja capaz de inibir o fator de crescimento vascular endotelial ${ }^{(27)}$ e restabelecer a barreira hemato-retiniana através da diminuição da sua permeabilidade ${ }^{(27-29)}$.

A associação de duas técnicas como a crioterapia e a injeção intravítrea de triancinolona, ou mesmo a utilização da triancinolona para o tratamento dos tumores vasoproliferativos não foi relatada na literatura. No caso relatado, acreditamos que a triancinolona, por suas propriedades antiangiogênicas, tenha funcionado como adjuvante no tratamento do tumor vasoproliferativo.

\section{CONCLUSÃO}

Até o presente momento, a associação de tumor vasoproliferativo e tuberculose ocular não foi relatada na literatura. $\mathrm{O}$ tratamento do tumor vasoproliferativo secundário com crioterapia e injeção intravítrea da triancionolona surge como nova opção terapêutica. Mais estudos são necessários para comprovar a eficácia deste tratamento.

\section{ABSTRACT}

Purpose: To describe a case of vasoproliferative tumor associated with presumed ocular tuberculosis treated with cryotherapy and intravitreal triamcinolone injection. Study Design/ Patient and Methods: Interventional case report. A 42-year-old female patient reported inflammation of the right eye 1 year ago, treated with oral prednisone for 30 days. She referred blurred vision in the right eye since childhood. Ophthalmologic examination showed 20/400 visual acuity in the right eye. Biomicroscopy and intraocular pressure were normal. Fundus examination showed vascularized elevated lesion, associated with serous retinal detachment and hard exudates at the inferior periphery. The lesion was surrounded by extensive area of hyperplastic retinal pigment epithelium. The macula showed attenuation of the foveal reflex. Ocular ultrasound showed a $2.25 \mathrm{~mm}$ height vascularized lesion. Serologic examination, hemogram and thorax RX were normal. PPD was considered strong reactor and tuberculosis was diagnosed after positive BK research. Considering the clinical aspects, the ocular diagnosis was vasoproliferative tumor associated with presumed ocular tuberculosis. Treatment with rifampicin, isoniazide and pyridoxine was started. We decided to treat the ocular tumor with cryotherapy and intravitreal triamcinolone injection $(4 \mathrm{mg} / \mathrm{ml})$. After 30 days, serous detachment was smaller and the tumor showed atrophic areas. Fluorescein angiography showed areas of vascular hyperfluorescence with slight extravasation and areas of blocked fluorescence due to RPE hyperplasia. Secondary vasoproliferative tumors are retinal glial proliferations and are associated with many ocular conditions that affect retina and choroid. Because of the great number of associated complications, with important visual loss, vasopro- 
liferative tumors should be treated at the moment of diagnosis. Treatment of choice is cryotherapy. Intravitreal triamcinolone can be used as adjuvant treatment because of its angiogenic properties. Until the present moment, no ocular tuberculosis and vasoproliferative tumor association were reported in the literature. Treatment of secondary vasoproliferative tumors with cryoterapy and intravitreal triamcinolone appears as a new therapeutic choice. Further studies are necessary to prove the efficacy of this association.

Keywords: Retinal neoplasms/diagnosis; Retinal neovascularization; Tuberculosis, ocular; Cryotherapy; Triamcinolone/ administration \& dosage; Case reports [Publication type]

\section{REFERÊNCIAS}

1. Shields JA, Decker WL, Sanborn GE, Augsburger JJ, Goldberg RE. Presumed acquired retinal hemangiomas. Ophthalmology. 1983;90(11):1292-300.

2. Shields CL, Shields JA, Barrett J, De Potter P. Vasoproliferative tumors of the ocular fundus. Classification and clinical manifestations in 103 patients. Arch Ophthalmol. 1995;113(5):615-23.

3. Smeets MH, Mooy CM, Baarsma GS, Mertens DE, Van Meurs JC. Histopathology of a vasoproliferative tumor of the ocular fundus. Retina. 1998;18(5):470-2.

4. Lafaut BA, Meire FM, Leys AM, Dralands G, De Laey JJ. Vasoproliferative retinal tumors associated with peripheral chorioretinal scars in presumed congenital toxoplasmosis. Graefes Arch Clin Exp Ophthalmol. 1999;237(12):1033-8.

5. McCabe CM, Mieler WF. Six-year follow-up of an idiopathic retinal vasoproliferative tumor. Arch Ophthalmol. 1996;114(5):617.

6. Campochiaro PA, Conway BP. Hemangiomalike masses of the retina. Arch Ophthalmol. 1988;106(10):1409-13.

7. Galinos SO, Smith TR, Brockhurst RJ. Angioma-like lesion in hemoglobin sickle cell disease. Ann Ophthalmol. 1979;11(10):1549-52.

8. Gottlieb F, Fammartino JJ, Stratford TP, Brockhurst RJ. Retinal angiomatous mass. A complication of retinal detachment surgery. Retina. 1984;4(3):152-7.

9. Laqua $\mathrm{H}$, Wessing A. Peripheral retinal telangiectasis in adults simulating a vascular tumor or melanoma. Ophthalmology. 1983;90(11):1284-91.

10. Irvine F, O'Donnell N, Kemp E, Lee WR. Retinal vasoproliferative tumors: surgical management and histological findings. Arch Ophthalmol. 2000; 118(4):563-9.

11. Heimann H, Bornfeld N, Vij O, Coupland SE, Bechrakis NE, Kellner U, Foerster MH. Vasoproliferative tumours of the retina. Br J Ophthalmol. 2000; 84(10):1162-9.
12. Welch RB. Von Hippel-Lindau disease: the recognition and treatment of early angiomatosis retinae and the use of cryosurgery as an adjunct to therapy. Trans Am Ophthalmol Soc. 1970;68:367-424.

13. Haik BG. Advanced Coats' disease. Trans Am Ophthalmol Soc. 1991;89: 371-476. Review.

14. Shields JA, Joffe L, Guibor P. Choroidal melanoma clinically simulating a retinal angioma. Am J Ophthalmol. 1978;85(1):67-71.

15. Bardenstein DS, Char DH, Irvine AR, Stone RD. Extramacular disciform lesions simulating uveal tumors. Ophthalmology. 1992;99(6):944-51.

16. Serracarbassa PD, Souza EC. Tumor vasoproliferativo idiopático da retina associado à hemorragia vítrea recidivante: relato de caso. Arq Bras Oftalmol. 2002;65(1):99-102.

17. Jampol LM, Goldbaum MH. Peripheral proliferative retinopathies. Surv Ophthalmol. 1980;25(1):1-14. Review.

18. Kreusel KM, Bornfeld N, Lommatzsch A, Wessing A, Foerster MH. Ruthenium106 brachytherapy for peripheral retinal capillary hemangioma. Ophthalmology. 1998;105(8):1386-92.

19. Madreperla SA, Hungerford JL, Plowman PN, Laganowski HC, Gregory PT. Choroidal hemangiomas: visual and anatomic results of treatment by photocoagulation or radiation therapy. Ophthalmology. 1997;104(11):1773-8; discussion 1779 .

20. Shields CL, Shields JA, Gunduz K, Freire JE, Mercado G. Radiation therapy for uveal malignant melanoma. Ophthalmic Surg Lasers. 1998;29(5):397-409. Review.

21. Barbezetto IA, Smith RT. Vasoproliferative tumor of the retina treated with PDT. Retina. 2003;23(4):565-7.

22. Schmidt-Erfurth UM, Kusserow C, Barbazetto IA, Laqua H. Benefits and complications of photodynamic therapy of papillary capillary hemangiomas. Ophthalmology. 2002;109(7):1256-66.

23. Florez J. Corticosteroids. In: Florez J, editor. Human pharmacology. Barcelona: Masson; 2003.

24. Machemer R, Sugita G, Tano Y. Treatment of intraocular proliferations with intravitreal steroids. Trans Am Ophthalmol Soc. 1979;77:171-80.

25. Jonas JB, Sofker A. Intraocular injection of crystalline cortisone as adjunctive treatment of diabetic macular edema. Am J Ophthalmol. 2001;132(3):425-7.

26. Bandi N, Kompella UB. Budesonide reduces vascular endothelial growth factor secretion and expression in airway (Calu-1) and alveolar (A549) epithelial cells. Eur J Pharmacol. 2001;425(2):109-16.

27. Wilson CA, Berkowitz BA, Sato Y, Ando N, Handa JT, de Juan E Jr. Treatment with intravitreal steroid reduces blood-retinal barrier breakdown due to retinal photocoagulation. Arch Ophthalmol. 1992;110(8):1155-9.

28. Penfold PL, Wen L, Madigan MC, King NJ, Provis JM. Modulation of permeability and adhesion molecule expression by human choroidal endothelial cells. Invest Ophthalmol Vis Sci. 2002;43(9):3125-30.

29. Sobrin L, D'Amico DJ. Controversies in intravitreal triamcinolone acetonide use. Int Ophhtalmol Clin. 2005;45(4):133-41.

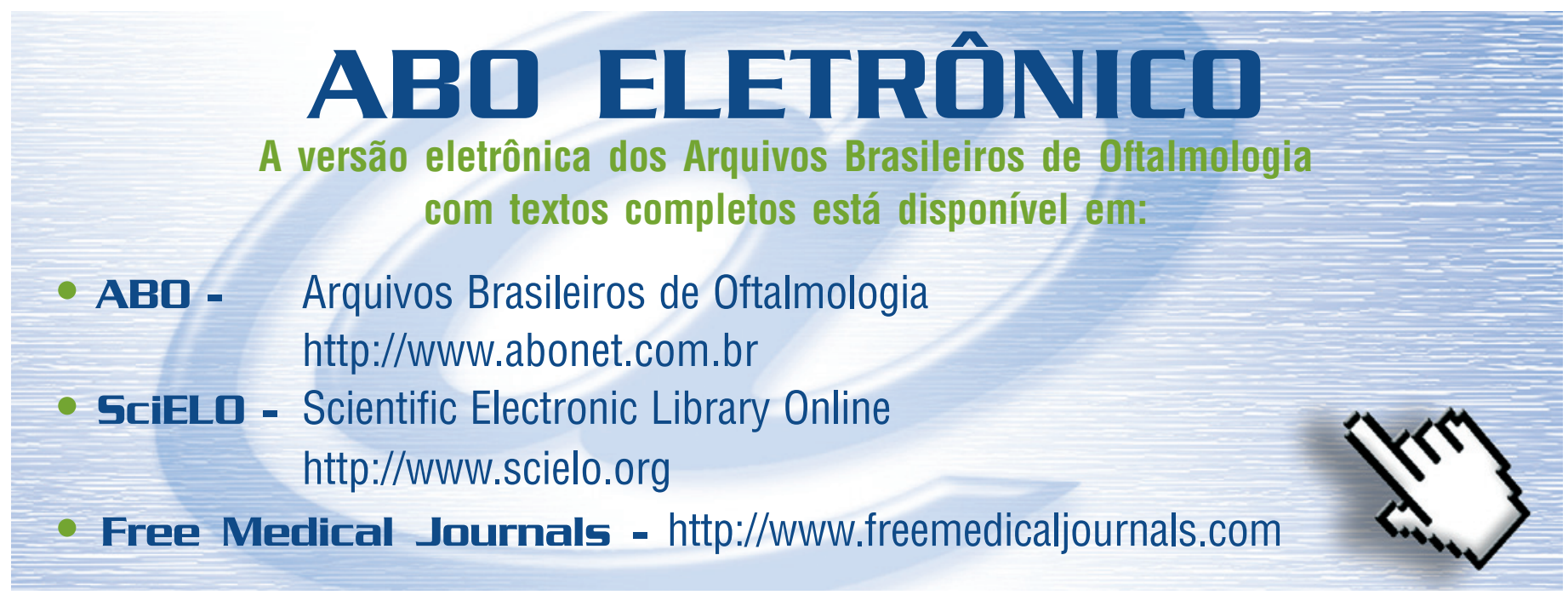

